\title{
The Contradiction of Drug Allergy in Patients with Cystic Fibrosis and Review of the Literature
}

\author{
Gokcen Dilsa Tugcu ${ }^{1}$, Nagehan Emiralioğlu ${ }^{1}$, Ebru Yalçın², Umit Sahiner ${ }^{2}$, Deniz Doğru ${ }^{2}$, \\ Bulent Sekerel $^{2}$, Uğur Özçelik ${ }^{2}$, Nural Kiper ${ }^{2}$, and Ozge Soyer ${ }^{3}$ \\ ${ }^{1}$ Hacettepe University Faculty of Medicine \\ ${ }^{2}$ Hacettepe University, İhsan Dogramacı Children's Hospital \\ ${ }^{3}$ Hacettepe University School of Medicine, Ihsan Dogramaci Children's Hospital
}

March 10, 2021

\begin{abstract}
Background: Cystic fibrosis (CF) is reported to be a risk factor for drug hypersensitivity. However, there is conflicting data about true prevalence of drug allergy in children with CF. Methods: The suspicious drug hypersensitivity reactions (DHR) of children with CF were enquired by European Network for Drug Allergy (ENDA) questionnaire and skin tests and/or drug provocation tests were performed according to established guidelines. Results: Two hundred and nineteen children (48.9\% boys; median [IQR] age, 8.4 years [4.8-12.4 years]) with cystic fibrosis were included in the study, from whom 22 patients with 24 suspected DHRs were evaluated. Most of the suspected DHRs were non-immediate $(\mathrm{n}=16,66.6 \%)$ type and the offending drugs were amoxicillin clavulanic acid $(\mathrm{n}=7)$, macrolides $(\mathrm{n}=4)$, trimethoprim sulfamethoxazole (TMP/SMX) $(\mathrm{n}=2)$, piperacillin tazobactam $(n=1)$, pancrelipase $(n=1)$ and ursodeoxycholic acid $(n=1)$. Eight $(33.3 \%)$ of the DHRs were classified as immediate [ceftriaxone $(n=2)$, ceftazidim $(n=2)$, meropenem $(n=1)$, ambisome $(n=2)$, vancomycin $(n=1)$ ]. The main presenting clinical presentations were maculopapular eruption (41.6\%) and urticaria (37.5\%), accompanied by angioedema (8.3\%), flushing (12.5\%) and vomiting $(8.3 \%)$. Nine skin tests (with beta-lactam protocol in 6 patients) and 24 DPTs were performed and none of the skin tests revealed a positive result, however 2 DPTs with TMP/SMX were positive. Conclusion: Actual drug allergy was demonstrated in 2 of 219 patients $(0.9 \%)$ with nonbeta-lactam antibiotics. These results conflict with previous researches that showed higher drug allergy rates but were consistent with some recent studies. Numerous and long-term use of multiple drugs during management of cystic fibrosis may contribute to tolerance development.
\end{abstract}

\section{The Contradiction of Drug Allergy in Patients with Cystic Fibrosis and}

\section{Review of the Literature}

Gokcen Dilsa Tugcu ${ }^{1 *}$, Nagehan Emiralioglu ${ }^{1 *}$, Ebru Yalcin ${ }^{1}$, Umit Murat Sahiner ${ }^{2}$, Deniz Dogru ${ }^{1}$, Bulent Enis Sekerel$^{2}$, Ugur Ozcelik ${ }^{1}$, Nural Kiper ${ }^{1}$, Ozge Soyer ${ }^{2}$

*contributed equally

${ }^{1}$ Hacettepe University, School of Medicine, Department of Pediatric Pulmonology

${ }^{2}$ Hacettepe University, School of Medicine, Department of Pediatric Allergy

Running Title: Drug Allergy in Cystic Fibrosis

Word count of abstract: 250

Word count of text: 2296

Number of tables and figures: 3 tables, 1 figure 
Conflict of interest: The authors declare that they have no conflict of interest

Financial support: There is no financial support

Corresponding Author:

ORCHID ID: 0000-0002-7444-251X

Özge SOYER, MD, Professor of Pediatrics

Hacettepe University Faculty of Medicine

Department of Pediatric Allergy

06100 Ankara Turkey

Phone: $+90-312-3051700$

E-mail: ozgeusoyer@gmail.com

\section{ABSTRACT}

Background: Cystic fibrosis (CF) is reported to be a risk factor for drug hypersensitivity. However, there is conflicting data about true prevalence of drug allergy in children with CF.

Methods: The suspicious drug hypersensitivity reactions (DHR) of children with CF were enquired by European Network for Drug Allergy (ENDA) questionnaire and skin tests and/or drug provocation tests were performed according to established guidelines.

Results: Two hundred and nineteen children (48.9\% boys; median [IQR] age, 8.4 years [4.8-12.4 years]) with cystic fibrosis were included in the study, from whom 22 patients with 24 suspected DHRs were evaluated. Most of the suspected DHRs were non-immediate $(\mathrm{n}=16,66.6 \%)$ type and the offending drugs were amoxicillin clavulanic acid $(n=7)$, macrolides $(n=4)$, trimethoprim sulfamethoxazole (TMP/SMX) $(n=2)$, piperacillin tazobactam $(n=1)$, pancrelipase $(n=1)$ and ursodeoxycholic acid $(n=1)$. Eight $(33.3 \%)$ of the DHRs were classified as immediate [ceftriaxone $(n=2)$, ceftazidim $(n=2)$, meropenem $(n=1)$, ambisome $(n=2)$, vancomy$\operatorname{cin}(\mathrm{n}=1)$ ]. The main presenting clinical presentations were maculopapular eruption $(41.6 \%)$ and urticaria $(37.5 \%)$, accompanied by angioedema (8.3\%), flushing (12.5\%) and vomiting (8.3\%). Nine skin tests (with beta-lactam protocol in 6 patients) and 24 DPTs were performed and none of the skin tests revealed a positive result, however 2 DPTs with TMP/SMX were positive.

Conclusion: Actual drug allergy was demonstrated in 2 of 219 patients (0.9\%) with nonbeta-lactam antibiotics. These results conflict with previous researches that showed higher drug allergy rates but were consistent with some recent studies. Numerous and long-term use of multiple drugs during management of cystic fibrosis may contribute to tolerance development.

Keywords: cystic fibrosis, drug allergy, drug hypersensitivity reactions, skin prick tests, drug provocation tests

Key points:

- Due to chronic nature of cystic fibrosis, the patients frequently exposed not only to antibiotics but also NSAIDs, proton pump inhibitors and others.

- Numerous and long-term use of multiple drugs during management of cystic fibrosis may contribute to tolerance development and protect patients from actual drug allergy.

- Non-beta lactam antibiotic hypersensitivity is important in patients with cystic fibrosis which requires diagnostic work up.

Introduction

Cystic fibrosis $(\mathrm{CF})$ is an inherited disease caused by mutations in the cystic fibrosis transmembrane conductance regulator protein which may affect all mucus producing organs in the body (1). The survival of patients 
with CF increased considerably by early diagnosis of disease, enhanced monitoring and earlier recognition of deterioration of organ function and detection and prompt treatment of airway infections (2).

Antibiotics are crucial components of CF management for prophylaxis and suppression of chronic infections and treatment of pulmonary exacerbations. Infections caused by pathogens such asStaphylococcus aureus and Pseudomonas aeruginosa require repetitive, aggressive, long term and broad-spectrum antibiotic treatments either inhaled, oral or parenteral, to preserve lung function (3). Besides, polypharmacy is common due to other systemic complications of the disease including pancreatic enzymes, mucolytics, ursodeoxycholic acid, proton pump inhibitors, non-steroidal anti-inflammatory drugs (NSAIDs), bisphosphonates and many others (4-6).

Frequent exposure to drugs, especially antibiotics, might cause the development of drug hypersensitivity reactions (DHRs), with both serious health and economic consequences. In patients with cystic fibrosis, the epidemiological studies concerning DHRs mainly pointed out beta-lactam antibiotics. Nevertheless, there is conflicting data that some studies reported a higher prevalence (28.8-61.9\%) (7-9) and others studies described similar results to general population (0.71-2.3\%) (10-12).

Therefore, in this study, we aimed to evaluate any suspected DHR in children with cystic fibrosis to determine the prevalence of confirmed DHRs and the culprit drugs and to analyze the risk factors that may facilitate the occurrence of drug allergies. Also, we reviewed the literature concerning the prevalence of DHRs in patients with cystic fibrosis to investigate the subject further.

Methods

We included children with cystic fibrosis followed at the Hacettepe University Pediatric Pulmonology Department. The study was performed in accordance with the protocol approved by the local ethical committee (Hacettepe University, GO 15/811), and all parents/guardians gave written informed consent.

We carried out a self-designed questionnaire survey concerning demographic factors [age at CF diagnosis, Cystic Fibrosis Transmembrane Conductance Regulator (CFTR) mutation results years of follow up, history of allergic diseases and DHRs, detailed data about chronically used medications (the onset and duration of use, administration route)] and reviewed medical records to note the hospitalizations due to lower respiratory tract infections and/or number of parenteral $\mathrm{AB}$ used within last year, average annual use of oral antibiotics, complications related with $\mathrm{CF}$ and chronic colonizations (Pseudomonas aeruginosa, Staphylococcus aureus ). In case of history or new occurrence of any suspicious DHR, the patients were evaluated by the pediatric allergy department.

\section{Study Measurements}

\section{Allergological work up}

If the drug HSR was immediate type, we performed skin testing with the culprit drug, histamine $(10 \mathrm{mg} / \mathrm{ml}$ of histamine phosphate, Allergopharma) as positive and $0.9 \%$ sterile saline as negative controls on the volar surface of forearm $(13,15)$. If skin prick test was negative, intradermal testing (IDT) with the culprit drug starting with minimum nonirritating concentration was done and assessed after 20 minutes (15). The test was considered as positive if a wheal of at least $3 \mathrm{~mm}$ greater in diameter than that of saline, accompanied by erythema, was formed. The evaluation of beta-lactam allergy was based on European Academy of Allergy and Clinical Immunology recommendations for skin prick test (SPT) and intradermal test (IDT) as amoxicillin and other semi-synthetic penicillins $(20 \mathrm{mg} / \mathrm{mL})$, cephalosporins $(20 \mathrm{mg} / \mathrm{mL})$, major (benzylpenicilloyl octaL-lysine, $0.04 \mathrm{mg} / \mathrm{mL}$ ) and minor (sodium benzylpenilloate, $0.5 \mathrm{mg} / \mathrm{mL}$ ) determinants (Diater S.A., Madrid, Spain) and benzylpenicillin $(10000 \mathrm{IU} / \mathrm{mL})$. In low-risk nonimmediate reactions with beta-lactams, direct provocation with the culprit drug without prior skin testing was preferred (14). In case of delayed HSRs, delayed reading of IDT was done if applicable (13).

Drug Provocation Tests 
The children underwent drug provocation tests (DPT) in case of mild or moderate index HSRs if the initial SPT and IDT revealed negative results. The patients were directly provoked with the culprit medication when there was no parenteral form of the drug proper for SPT/IDT (e.g.,NSAIDs) or there were no standard ST protocols established (e.g. clarithromycin) (16). Oral form of the drug was preferred for DPT and the doses and intervals were determined according to the established protocols (17). The patient was administered the age- and weight-adjusted total daily dose in four or five divided doses $(14,17)$. The positive DPT was defined as the development of any objective physical finding of a HSR concerning cutaneous (urticaria, maculopapular rash, flushing which occurred during the DPT or within $48 \mathrm{hrs}$ ), respiratory, gastrointestinal, and cardiovascular system $(13,14)$. For non-immediate reactions, one day protocol was performed to protect patients from longer exposure to antimicrobials.

\section{Statistical analysis}

Statistical analyses were done using SPSS software version 21 (SPSS, Inc). The demographic factors of $\mathrm{CF}$ patients and reactions were described as frequencies for categorical variables. The values were given as medians (interquartile ranges) for data that did not show a normal distribution.

\section{Results}

Two hundred and nineteen children (48.9\% boys; median [IQR] age, 8.4 years [4.8-12.4 years]) with cystic fibrosis were included in the study. The median age at diagnosis of CF was 3 (2-6) months; the duration of follow up was 5.8 (3.3-10.1) years (Table 1). Seventy-six (24.7\%) patients carried at least one del F508 mutation whereas nearly half of the children were demonstrated to have other mutations. Some of the children were colonized chronically with Pseudomonas aeruginosa $(\mathrm{n}=36,16.5 \%)$ and Staphylococcus aureus $(\mathrm{n}=56$, $25.6 \%)$. Nearly all patients (99\%) were using medications chronically (Table 2), 214 (97.7\%) of them received at least one course of antibiotics within last year. One fourth of the patients had been administered at least one course of parenteral antibiotic treatment in hospital in the last 1 year.

Twenty-two patients were referred to the pediatric allergy department with 24 suspected drug related hypersensitivity reactions (Figure 1). The route of administration of these drugs were mainly oral $(\mathrm{n}=13)$ than parenteral $(\mathrm{n}=9)$. Most of the suspected DHRs were non-immediate $(\mathrm{n}=16,66.6 \%)$ type and the offending drugs were amoxicillin clavulanic acid $(\mathrm{n}=7)$, macrolides $(\mathrm{n}=4)$, trimethoprim sulfamethoxazole (TMP/SMX) $(n=2)$, piperacillin tazobactam $(n=1)$, pancrelipase $(n=1)$ and ursodeoxycholic acid $(n=1)$. Eight $(33.3 \%)$ of the DHRs were classified as immediate [ceftriaxone $(n=2)$, ceftazidime $(n=2)$, meropenem $(n=1)$, ambisome $(\mathrm{n}=2)$, vancomycin $(\mathrm{n}=1)]$. The presenting clinical presentations were maculopapular eruption $(41.6 \%)$ and urticaria (37.5\%), accompanied by angioedema (8.3\%), flushing (12.5\%) and vomiting (8.3\%). None of the patients experienced anaphylaxis or severe cutaneous adverse reactions.

We performed 9 skin tests (with beta-lactam protocol in 6 patients) and 24 DPTs to determine the actual drug allergy (Figure 1). None of the skin tests revealed a positive result, however 2 DPTs with TMP/SMX were positive. Twelve-year-old boy (CF mutation Q720X/Q720X) developed generalized maculopapular eruption on second day of TMP/SMX treatment, after 3 to 4 hours of ultimate dose which resolved within two days of cessation of drug at ages of seven and eleven. The patient presented with maculopapular eruption again after six hours of DPT with TMP/SMX. Nine-year-old girl (CF mutation E92K) experienced generalized urticaria and angioedema of face, 4 hours after last dose of TMP/SMX, on day 3 of treatment. The HSR resolved with administration of antihistamine and systemic corticosteroid within 6 hours. The skin testing with TMP/SMX (0.8 mg/ ml) (18) demonstrated to be negative, nevertheless the DPT confirmed generalized urticaria had occurred two hours after administration of TMP/SMX, suggesting that the TMP/SMX was the culprit drug.

In summary, actual drug allergy was demonstrated in 2 of 219 patients (0.9\%) with nonbeta-lactam antibiotics after diagnostic work up for DHRs. Due to low prevalence of actual drug allergy in our cohort, we were not able to define any risk factor for occurrence of DHRs in children with cystic fibrosis.

\section{Discussion}


In this study, $10.04 \%$ of our patients with cystic fibrosis underwent diagnostic work up due to symptoms suggesting any drug related hypersensitivity reaction and we confirmed drug allergy in $0.9 \%$ of the study population. Unlike previous studies, none of the children exhibited beta-lactam hypersensitivity, instead a non-beta lactam antibiotic, TMP/SMX was the culprit agent responsible for DHRs.

There is limited data concerning prevalence of confirmed DHRs in general population. Rebelo et al demonstrated parentally-reported drug allergy prevalence as $4.6 \%$ in children and diagnostic work up revealed confirmed drug allergy in $0.2 \%$ of the study population (19). In a population based study concerning school aged children, the true frequency of immediate type drug hypersensitivity was shown as $0.11 \%(20)$. In the present study, the prevalence of DHRs was similar to general population, however, lower than the prevalence of DHRs compared to some previous studies (28.8-61.9\%) $(7-9,21)$.

Since there is contradictory results about the frequency of DHRs in the literature, we summarized these studies in Table 3 to make a comparison. In earlier studies, the prevalence of DHRs in patients with CF ranged between 28.8-61.9\% (7-9, 21, 22). There might be a number of comments for this. The majority of patients with cystic fibrosis included were only adults or adults and children. Burrows et al demonstrated that the age of the patients with CF who experienced a DHR was older and a higher proportion of patients older than 25 years had DHRs compared with younger patients. Further, higher number of antibiotics courses were not associated only with higher risk of antibiotic allergy but also with allergy to more than one antibiotic (22). However, Roehmel et al showed that DHRs were drug-specific and dependent on cumulative annual dose (7). The definitions of DHRs might not fit all criteria for DHRs defined in guidelines. Pleasant et al described the DHRs as rash and drug induced fever in their study (8). Besides, the clinical presentations of DHRs were not detailed in most of these studies. Probably the most important problem in these studies was that the guidelines defined allergy assessment steps were not followed, and most studies were retrospective and based on questionnaires and / or medical records. Nevertheless, skin tests and DPTs were not performed for confirmation of true drug allergy $(7-9,21,22)$. It is well-known that, minority of suspected DHRs were proved to be accurate drug allergy (19).

It is encouraging that our results are consistent with that found by Matar (11), Braun (12) and Caimmi (10) et al ranging between 0.71-2.3\% . Caimmi et al. conducted skin tests and DPTs on $14 \%$ of 171 patients with suggestive DHR due to beta-lactam antibiotics and $2.3 \%$ of the study cohort was diagnosed as immediate type beta-lactam allergy (10). The reason for higher frequency of DHRs in cystic fibrosis has been argued that repetitive antibiotic administrations may result in increased risk of sensitization $(7,21,22)$. Rather, recurrent exposure to drugs, especially by courses longer than applied to everyone might contribute to development of tolerance. Unfortunately, our patients also used antibiotics frequently. One fourth of our patients were hospitalized due to pneumonia which were treated by parenteral antibiotics within last year. Although we did not have the data related to the total number of antibiotic courses up to child's age; in the last year, nearly all consumed antibiotics at least once and half of the cases used antibiotics more than twice.

One unanticipated finding was that there were no cases with beta-lactam allergy, besides two patients were diagnosed as TMP/SMX allergy (0.9\%). Recently, in a population of 601 patients with cystic fibrosis, the prevalence of TMP/SMX hypersensitivity was indicated as $0.49 \%$ and found as the second class of antibiotics responsible for DHRs (12). Self-reported sulfa allergy ranged between $0.07-2.7 \%$ in different populations (23-25). Increased antigenicity to sulfamethoxazole in sulfamethoxazole-hypersensitive patients with cystic fibrosis was demonstrated. T cells from sulfamethoxazole-hypersensitive patients with cystic fibrosis were stimulated with oxidative metabolites of sulfamethoxazole more than sulfamethoxazole-hypersensitive patients without cystic fibrosis in terms of higher secretion of IFN-g, IL-6, and IL-10. Additionally, recurrent infections might enhance sulfamethoxazole metabolite formation, therefore increased protein adduct formation associated with the stimulation of sulfamethoxazole-responsive T cells (26). In view of the results of these studies, physicians dealing with cystic fibrosis should be more vigilant about DHRs due to TMP/SMX HRs which was an understudied area.

As far as we know, this is the first study which focused on prevalence of any drug hypersensitivity reaction in patients with cystic fibrosis. In the present work, we extensively inquired patients and reviewed medical 
records for DHRs related to drugs other than beta-lactam and non-beta lactam antibiotics. Due to chronic nature of cystic fibrosis, the patients frequently exposed not only to antibiotics but also NSAIDs, proton pump inhibitors and others (6). Use of the offending drug or cross-reactive ones in chronic or repetitive treatment schedules enhance the risk for DHRs. Actual drug allergy was shown to be more frequent in patients with chronic diseases $(27,28)$. However, we were unable to demonstrate hypersensitivity to drugs other than antibiotics.

There are some limitations of this study. We did not have detailed information about the classes of antibiotics and NSAIDs and duration of treatment courses used during lifetime. The study had both retrospective and prospective aspects that some of diagnostic tests might not have been done with optimum timing.

As adverse drug reactions accumulate over time, the physicians will try to manage their patients with $\mathrm{CF}$ with limited number of treatment options. Therefore, it is crucial to assess cases according to established diagnostic protocols. Numerous and long-term use of multiple drugs during management of cystic fibrosis may contribute to tolerance development and protect patients from actual drug allergy.

\section{References}

1. Villanueva G, Marceniuk G, Murphy MS, Walshaw M, Cosulich R, Guideline C. Diagnosis and management of cystic fibrosis: summary of NICE guidance. Bmj. 2017;359:j4574.

2. Bell SC, Mall MA, Gutierrez H, Macek M, Madge S, Davies JC, et al. The future of cystic fibrosis care: a global perspective. The Lancet Respiratory medicine. 2020;8(1):65-124.

3. Castellani C, Duff AJA, Bell SC, Heijerman HGM, Munck A, Ratjen F, et al. ECFS best practice guidelines: the 2018 revision. Journal of cystic fibrosis : official journal of the European Cystic Fibrosis Society. 2018;17(2):153-78.

4. Konstan MW, VanDevanter DR, Sawicki GS, Pasta DJ, Foreman AJ, Neiman EA, et al. Association of High-Dose Ibuprofen Use, Lung Function Decline, and Long-Term Survival in Children with Cystic Fibrosis. Annals of the American Thoracic Society. 2018;15(4):485-93.

5. McCrory BE, Harper HN, McPhail GL. Use and Incidence of Adverse Effects of Proton Pump Inhibitors in Patients with Cystic Fibrosis. Pharmacotherapy. 2018.

6. Dogru D, Cakir E, Sismanlar T, Cobanoglu N, Pekcan S, Cinel G, et al. Cystic fibrosis in Turkey: First data from the national registry. Pediatric pulmonology. 2020;55(2):541-8.

7. Roehmel JF, Schwarz C, Mehl A, Stock P, Staab D. Hypersensitivity to antibiotics in patients with cystic fibrosis. Journal of cystic fibrosis : official journal of the European Cystic Fibrosis Society. 2014;13(2):205-11.

8. Pleasants RA, Walker TR, Samuelson WM. Allergic reactions to parenteral beta-lactam antibiotics in patients with cystic fibrosis. Chest. 1994;106(4):1124-8.

9. Koch C, Hjelt K, Pedersen SS, Jensen ET, Jensen T, Lanng S, et al. Retrospective clinical study of hypersensitivity reactions to aztreonam and six other beta-lactam antibiotics in cystic fibrosis patients receiving multiple treatment courses. Reviews of infectious diseases. 1991;13 Suppl 7:S608-11.

10. Caimmi S, Sanfiorenzo C, Caimmi D, Bousquet PJ, Chiron R, Demoly P. Comprehensive allergy workup is mandatory in cystic fibrosis patients who report a history suggestive of drug allergy to beta-lactam antibiotics. Clinical and translational allergy. 2012;2(1):10.

11. Matar R, Le Bourgeois M, Scheinmann P, de Blic J, Ponvert C. Beta-lactam hypersensitivity in children with cystic fibrosis: a study in a specialized pediatric center for cystic fibrosis and drug allergy. Pediatric allergy and immunology : official publication of the European Society of Pediatric Allergy and Immunology. 2014;25(1):88-93.

12. Braun C, Reix P, Durieu I, Nove-Josserand R, Durupt S, Ohlmann C, et al. The diagnosis of hypersensitivity to antibiotics is rarely confirmed by allergy work-up in cystic fibrosis patients. Pediatric aller- 
gy and immunology : official publication of the European Society of Pediatric Allergy and Immunology. 2020;31(4):396-404.

13. Demoly P, Adkinson NF, Brockow K, Castells M, Chiriac AM, Greenberger PA, et al. International Consensus on drug allergy. Allergy. 2014;69(4):420-37.

14. Romano A, Atanaskovic-Markovic M, Barbaud A, Bircher AJ, Brockow K, Caubet JC, et al. Towards a more precise diagnosis of hypersensitivity to beta-lactams - an EAACI position paper. Allergy. 2020;75(6):1300-15.

15. Brockow K, Garvey LH, Aberer W, Atanaskovic-Markovic M, Barbaud A, Bilo MB, et al. Skin test concentrations for systemically administered drugs - an ENDA/EAACI Drug Allergy Interest Group position paper. Allergy. 2013;68(6):702-12.

16. Cavkaytar O, Karaatmaca B, Yilmaz EA, Sekerel BE, Soyer O. Testing for clarithromycin hypersensitivity: A diagnostic challenge in childhood. The journal of allergy and clinical immunology In practice. 2016;4(2):330-2 e1.

17. Aberer W, Bircher A, Romano A, Blanca M, Campi P, Fernandez J, et al. Drug provocation testing in the diagnosis of drug hypersensitivity reactions: general considerations. Allergy. 2003;58(9):854-63.

18. Kuyucu S, Mori F, Atanaskovic-Markovic M, Caubet JC, Terreehorst I, Gomes E, et al. Hypersensitivity reactions to non-betalactam antibiotics in children: an extensive review. Pediatric allergy and immunology : official publication of the European Society of Pediatric Allergy and Immunology. 2014;25(6):534-43.

19. Rebelo Gomes E, Fonseca J, Araujo L, Demoly P. Drug allergy claims in children: from self-reporting to confirmed diagnosis. Clinical and experimental allergy : journal of the British Society for Allergy and Clinical Immunology. 2008;38(1):191-8.

20. Erkocoglu M, Kaya A, Civelek E, Ozcan C, Cakir B, Akan A, et al. Prevalence of confirmed immediate type drug hypersensitivity reactions among school children. Pediatric allergy and immunology : official publication of the European Society of Pediatric Allergy and Immunology. 2013;24(2):160-7.

21. Wills R, Henry RL, Francis JL. Antibiotic hypersensitivity reactions in cystic fibrosis. Journal of paediatrics and child health. 1998;34(4):325-9.

22. Burrows JA, Nissen LM, Kirkpatrick CM, Bell SC. Beta-lactam allergy in adults with cystic fibrosis. Journal of cystic fibrosis : official journal of the European Cystic Fibrosis Society. 2007;6(4):297-303.

23. Macy E, Poon KYT. Self-reported antibiotic allergy incidence and prevalence: age and sex effects. The American journal of medicine. 2009;122(8):778 e1-7.

24. Orhan F, Karakas T, Cakir M, Akkol N, Bahat E, Sonmez FM, et al. Parental-reported drug allergy in 6- to 9-yr-old urban schoolchildren. Pediatric allergy and immunology : official publication of the European Society of Pediatric Allergy and Immunology. 2008;19(1):82-5.

25. Lange L, Koningsbruggen SV, Rietschel E. Questionnaire-based survey of lifetime-prevalence and character of allergic drug reactions in German children. Pediatric allergy and immunology : official publication of the European Society of Pediatric Allergy and Immunology. 2008;19(7):634-8.

26. Elsheikh A, Castrejon L, Lavergne SN, Whitaker P, Monshi M, Callan H, et al. Enhanced antigenicity leads to altered immunogenicity in sulfamethoxazole-hypersensitive patients with cystic fibrosis. The Journal of allergy and clinical immunology. 2011;127(6):1543-51 e3.

27. Tugcu GD, Cavkaytar O, Sekerel BE, Sackesen C, Kalayci O, Tuncer A, et al. Actual drug allergy during childhood: Five years' experience at a tertiary referral centre. Allergologia et immunopathologia. 2015;43(6):571-8.

28. Greenberger PA. Drug allergy. Allergy and asthma proceedings. 2019;40(6):474-9. 


\section{Hosted file}

Table 1. Demographic characteristics of the CF cohort.odt available at https://authorea.com/ users/369107/articles/512967-the-contradiction-of-drug-allergy-in-patients-with-cysticfibrosis-and-review-of-the-literature

\section{Hosted file}

Table 2. Chronically used medications.odt available at https://authorea.com/users/369107/ articles/512967-the-contradiction-of-drug-allergy-in-patients-with-cystic-fibrosis-andreview-of-the-literature

\section{Hosted file}

Table 3. Summary of studies concerning prevalence of DHRs in CF.odt available at https: //authorea.com/users/369107/articles/512967-the-contradiction-of-drug-allergy-inpatients-with-cystic-fibrosis-and-review-of-the-literature

\section{Hosted file}

Figure 1. Flow chart of the study.pptx available at https://authorea.com/users/369107/ articles/512967-the-contradiction-of-drug-allergy-in-patients-with-cystic-fibrosisand-review-of-the-literature 